\title{
MODELING GRANULAR CRUSHING IN RING SHEAR TESTS: EXPERIMENTAL AND NUMERICAL ANALYSES
}

\author{
Sebastian Lobo-Guerreroi) and Luis E. VALLEJo ${ }^{\mathrm{ii})}$
}

\begin{abstract}
A fault consists of a zone of heavily fragmented granular rock (gouge), which is confined between two rough walls made of fractured rock. The granular gouge is the result of previous fracturing of the wall rock by the combined effect of compressive and shear stresses. Through time, the granular fault gouge will experience various episodes of further fragmentation (crushing) as a result of the mobilization by shear of the fault walls. The evolution of crushing in a simulated gouge material was studied using laboratory ring shear tests and DEM ring shear simulations. The laboratory ring shear tests were developed using sugar as a weak granular material. It was found that the residual friction coefficient of this material maintained a constant value regardless of the severe degradation of the particles. This degradation was induced by increasing the angular deformation or increasing the applied vertical stress. Moreover, it was found that the grain size distribution of the original uniform material evolved toward a fractal distribution of sizes. The results from the DEM simulations confirmed those from the laboratory tests and provided also a visualization of the evolution of crushing. Event though originally DEM does not consider particle breakage, this was allowed by replacing particles fulfilling a predefined tensile failure criterion with an equivalent group of smaller particles.
\end{abstract}

Key words: discrete element method, micro mechanics, particle crushing, ring shear test, strength (IGC: D5/D6)

\section{INTRODUCTION}

The continuous movement of the wall rocks in some natural faults generates a finely crushed rock layer called fault gouge. The behavior of these faults is strongly influenced by the mechanical properties of this material. Previous research has shown that particle crushing results as a consequence of increments on stresses and induced deformations inside faults, and the grain size distribution of the fault gouge material evolves toward a fractal distribution of particle sizes (Sammis, 1997). Previous research has also shown that changes in the grain size distribution of a granular material are associated with changes in its hydraulic conductivity and deformation characteristics (Vallejo, 2003). Therefore, is important to understand how different properties of the fault gouge change as a consequence of granular crushing and how these changes affect the satiability of faults. This study reports the results obtained in laboratory and simulated ring shear tests developed in order to analyze and visualize the evolution of crushing in a continuous shearing zone. Laboratory ring shear tests were conducted using a weak granular material (sugar), while the computer simulations were developed using a modified version of the discrete element method (DEM).

This study is a continuation of the work presented by
Lobo-Guerrero and Vallejo (2005). The previous study focused on the evolution of granular crushing in the direct shear apparatus. The granular samples during the direct shear testing were subjected to a maximum horizontal deformation of $9 \mathrm{~mm}$ during the actual test and $5 \mathrm{~mm}$ in the DEM simulation of the test (these deformations correspond to shear strains equal to $43 \%$ and $17 \%$ respectively). The present study focused on the evolution of crushing at large values of deformation as measured in the ring shear test. The maximum shear strain induced in the three-dimensional actual ring shear tests was equal to $5340 \%$. In the two-dimensional DEM simulations of the ring shear test the samples were subjected to a maximum shear strain equal to $250 \%$. The purpose of the 2-D DEM simulations was to understand and visualize how crushing develops in granular material subjected to large values of shear strain similar but not equal to the ones induced by actual ring shear tests. By conducting the ring shear tests (actual and simulated), important aspects such as the mobilized shear strength at large values of strain, the possibility of reaching a critical state where granular crushing stops, and the development of a fractal grain size distribution as a result of crushing taking place at large shear deformations were studied.

i) Ph.D. Student, Department of Civil and Environmental Engineering, University of Pittsburgh, Pittsburgh, PA, USA (sel2@pitt.edu).

ii) Professor, ditto (vallejo@ civ.pitt.edu).

The manuscript for this paper was received for review on February 15, 2005; approved on January 13, 2006.

Written discussions on this paper should be submitted before November 1, 2006 to the Japanese Geotechnical Society, 4-38-2, Sengoku, Bunkyo-ku, Tokyo 112-0011, Japan. Upon request the closing date may be extended one month. 


\section{PREVIOUS WORK}

Studying crushing of granular materials has always been limited by the machines needed to develop the considerable loads that can lead to grain fragmentation. One way to sidestep this is to use standard geotechnical equipment with weak materials (Mandl et al., 1977; McDowell and Humphreys, 2002; McDowell and Khan, 2003). Previous researchers have reported results from ring shear tests on weak granular materials such as carbonate sand (Luzzani and Coop, 2002; Coop et al., 2004). Two interesting features have been revealed concerning the behavior of crushable granular materials: a) the grain size distribution of the original material evolves toward an stable fractal distribution as a consequence of particle breakage; however, this stable fractal distribution of sizes depends on the magnitude of induced stresses and the initial grading of the sample; b) The mobilized angle of shearing resistance does not significantly change regardless the severe degradation of the material. In this way, ring shear tests conducted on weak granular materials have shown to be a valuable tool in order to understand the evolution of crushing.

One disadvantage of using ring shear tests in the laboratory is that they do not easily allow a visualization of the crushing process. Numerical simulations in the form of the Discrete Element Method (DEM) have been used in order to overcome this constrain. Since the original DEM developed by Cundall and Strack (1979) does not consider particle breakage, different solutions have been proposed and programmed using DEM codes. The first solution to this problem is to develop several ring shear simulations considering different grain size distributions. In this way, each grain size distribution represents a different state in the evolution of crushing (Morgan, 1999; Morgan and Boettcher, 1999). The second solution is to treat each granular particle inside the ring shear as a porous agglomerate built by bonding smaller particles, which is defined as a cluster (Jensen et al., 2001). Following certain criteria, this cluster can fully or partially disaggregate during the simulation. A third solution to this problem consists on replacing the particles that are fulfilling a predefined failure criterion with an equivalent group of smaller particles (Lang, 2002). This study uses the third solution and a simplified tensile failure criterion that can be easily implemented on DEM. This paper also presents and compares the results obtained using ring shear tests conducted on sugar and the results obtained using DEM.

\section{EXPERIMENTAL ANALYSIS}

Ring shear tests were carried out using sugar in a standard Bromhead type apparatus (Fig. 1). The surface of the platens of the ring shear apparatus applying the normal and shear stresses to the samples were rough. These surfaces had the form of a zigzag pattern with a height equal to $1 \mathrm{~mm}$ and a distance between peaks equal to $1 \mathrm{~mm}$. During the testing program the humidity and air temperature were carefully controlled, so the sugar used did not experience any visible change in structure. The sugar grains tested had an average diameter equal to $1.015 \mathrm{~mm}$ (material passing sieve No. 16 and retained in sieve No. 20). The coefficient of uniformity of the samples tested was equal to 1.2 . The specific gravity, $G_{\mathrm{s}}$, was equal to 1.5 . The maximum and minimum void ratios were equal to 0.68 and 0.48 respectively. The void ratio at the beginning of the tests was equal to 0.65 . The natural angle of repose (friction angle) was equal to $40^{\circ}$. The coefficient of compressibility of this sugar as determined from the slope of the normal compression line from an oedometer test is equal to 0.37 (Lobo-Guerrero and Vallejo, 2004). The tested samples had an outside radius of $5 \mathrm{~cm}$ and an inside radius of $3.5 \mathrm{~cm}$. The samples height was equal to $5 \mathrm{~mm}$. They were prepared by placing the sugar inside the ring shear by using the pluviation method. Thus, the samples had a loose state at the beginning of the tests and crushing of the sugar grains was avoided during the preparation of the samples. Figure 1 shows an example of the initial state of the samples. As shown on these pictures, the original particles had a prismatic rectangular shape. The height of a grain was around $1.7 \mathrm{~mm}$, the width and thickness both measured $1 \mathrm{~mm}$. The cap shown in Fig. 1 was placed on top of each sample after its preparation. Each sample was placed on the ring shear apparatus and was subjected to a vertical stress. Three different vertical stresses were used in the testing program: $198 \mathrm{kPa}, 296 \mathrm{kPa}$, and $394 \mathrm{kPa}$. The angular velocity of the upper part of the ring shear had a constant value of 1.2 degree/minute during the tests.

\section{Mobilized Friction Coefficient}

Figure 2 shows the curves relating the mobilized friction coefficient $(\tau / \sigma)$ and the level of horizontal deformation for the normal stresses used on the tests (horizontal displacement $=$ [average radius] $[$ angular displacement in rads]). As is explained later in this paper, it was found that the sugar achieved an almost constant grading before completing one revolution (horizontal displacement of $26.7 \mathrm{~cm}$ ), and very small crushing was expected to occur after this deformation. Figure 2 indicates little variation of the mobilized friction coefficient with respect to the normal stresses and the induced deformations. It can be observed that a constant friction coefficient for all the vertical stresses varied between 0.55 and 0.6 (corresponding to angles of shearing resistance between $28.8^{\circ}$ and $31^{\circ}$ ). An average value for this constant friction coefficient is 0.575 that corresponds with an angle of shearing resistance of $29.9^{\circ}$. This angle of shear resistance is considerably lower than the angle of friction before crushing $\left(40^{\circ}\right)$ measured with the angle of repose.

\section{The Evolution of Crushing}

Figure 3 shows the average size compositions of the three samples subjected to vertical stress of $198 \mathrm{kPa}$, $296 \mathrm{kPa}$, and $394 \mathrm{kPa}$ after one revolution in the ring shear. Figure 3 also shows how the original uniform 

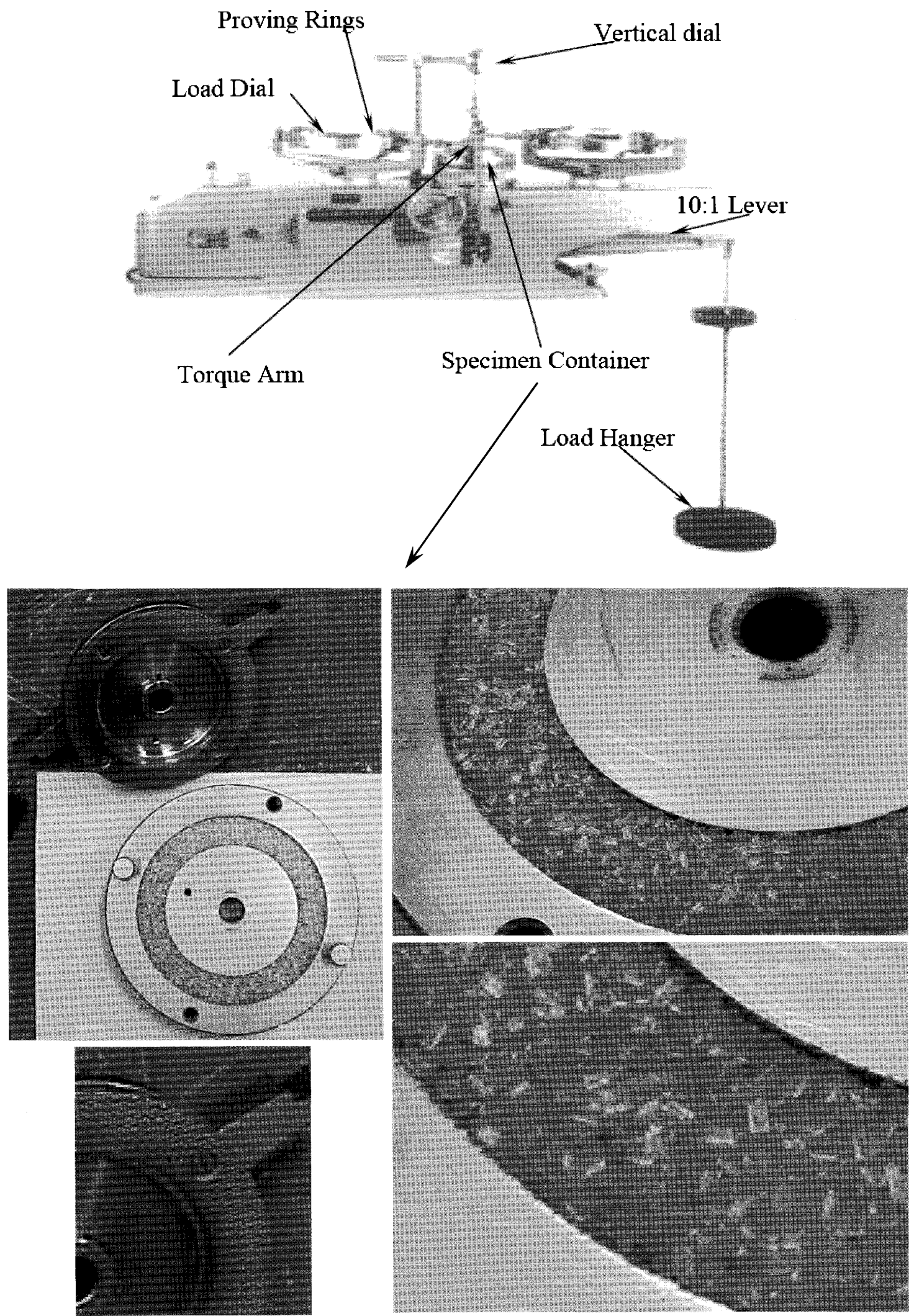

Fig. 1. Description of the ring shear apparatus with sugar samples 


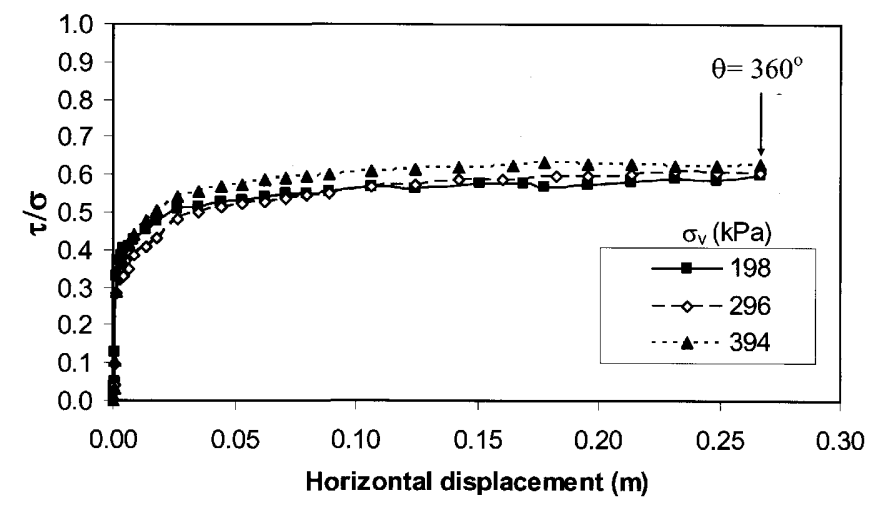

Fig. 2. Friction coefficient vs. horizontal deformation, laboratory results

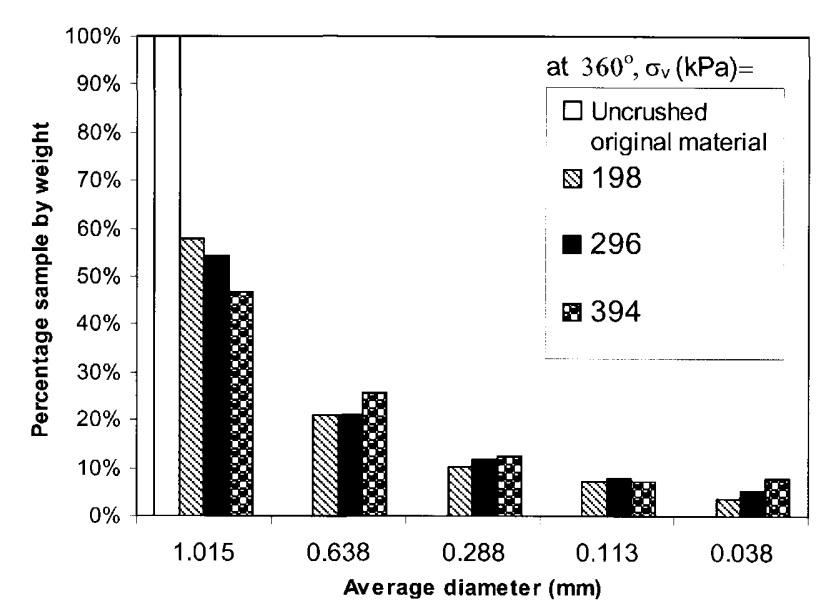

Fig. 3. Particle size distribution of the samples after one revolution on the laboratory ring shear

material became a well graded mixture of sizes after the tests. It can be noted that the amount of crushing increased with the magnitude of the applied vertical stress. A comparison of Figs. 2 and 3 shows that even though the samples had different level of crushing they all exhibited a constant friction coefficient. This is in agreement with the laboratory results recently reported by Coop et al. (2004).

Figure 3 does not provide any information regarding the evolution of crushing inside the samples with respect to the horizontal deformation since it only shows the final configuration of the sugar particles. Thus, four more tests were conducted on the ring shear apparatus. The four tests had vertical stresses of $296 \mathrm{kPa}$, but where ended at different values of angular deformation $\left(0^{\circ}, 90^{\circ}\right.$, $\left.180^{\circ}, 270^{\circ}\right)$. Sieve and photographic analyses were conducted after these tests. Figure 4 shows the average size compositions of the four samples at the end of each test (the results obtained at $360^{\circ}$ were also included in this figure). It can be observed that most of the crushing was produced before an angular deformation of $90^{\circ}$. Moreover, there was only a small amount of crushing (almost 3\%) between angular deformations of $270^{\circ}$ and $360^{\circ}$. It can be concluded that the sample at $360^{\circ}$ tended to reach a stable grain size distribution where almost no

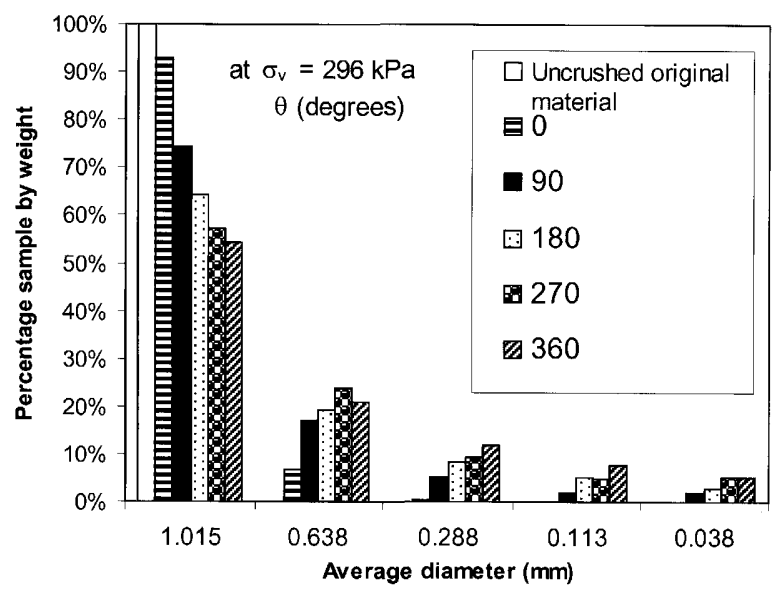

Fig. 4. Particle size distribution of the laboratory samples at $\sigma_{v}=296$ $\mathbf{k P a}$

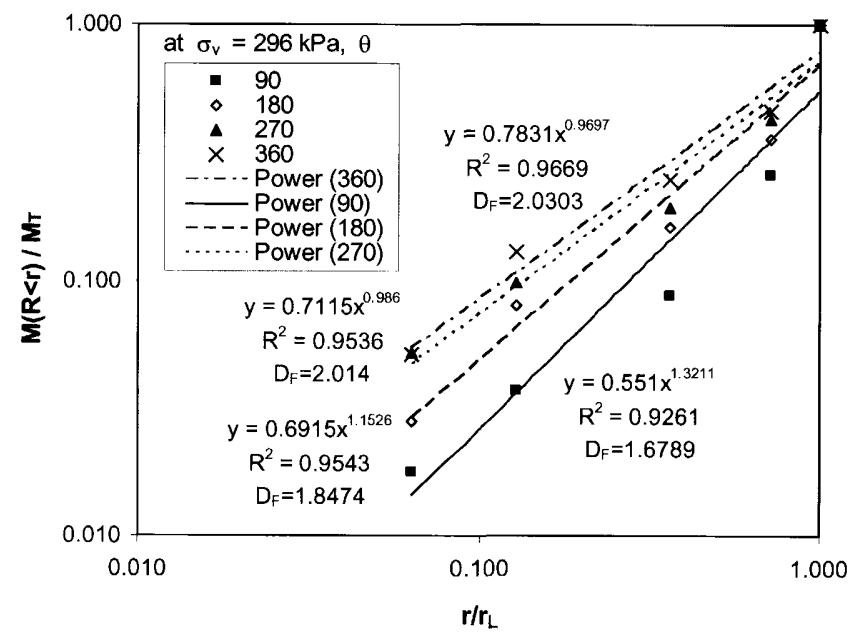

Fig. 5. Fragmentation fractal dimensions of the laboratory samples at $\sigma_{v}=296 \mathrm{kPa}$

crushing was expected to occur.

Using the results from the sieve analyses, the fragmentation fractal dimension of the samples were calculated using the following equation (Tyler and Wheatcraft, 1992):

$$
\frac{M(R<r)}{M_{\mathrm{T}}}=\left(\frac{r}{r_{\mathrm{L}}}\right)^{3-D_{\mathrm{f}}}
$$

Where $M(R<r)$ is the cumulative mass of the particles with size $R$ smaller than a given comparative size $r . M_{\mathrm{T}}$ represents the total mass of particles, $r$ is the sieve size opening, and $r_{\mathrm{L}}$ is the maximum particle size as defined by the largest sieve size opening used in the analysis. $D_{\mathrm{f}}$ is the fragmentation fractal dimension. Figure 5 shows the obtained results after applying Eq. (1) to the results of the sieve analyses. Also, power regression lines were added to the laboratory data and the fragmentation fractal dimensions of the samples were calculated. It can be noted that the fragmentation fractal dimensions of the samples corresponding to angular deformations of $270^{\circ}$ and $360^{\circ}$ were similar (2.014 and 2.03) which confirms 


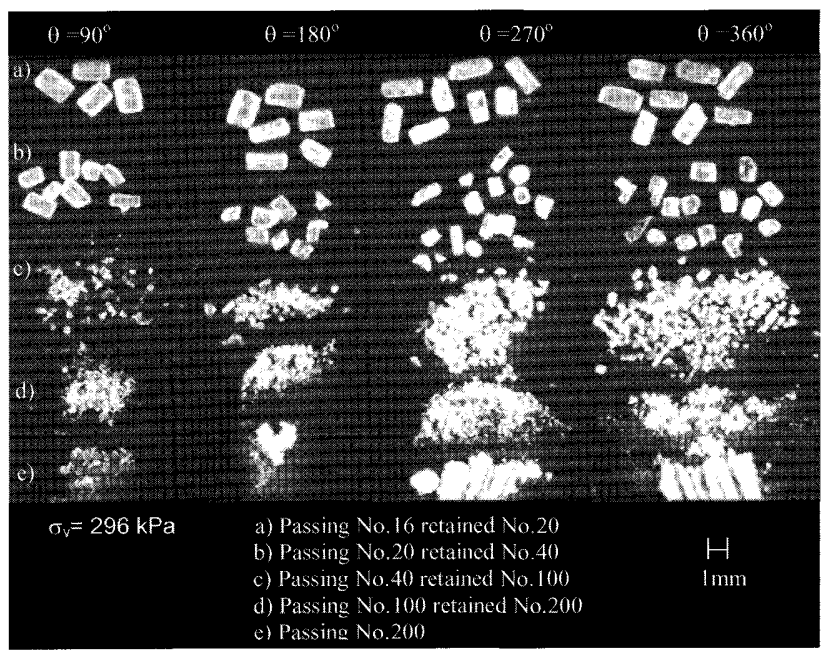

Fig. 6. Photograph of particles from the laboratory samples at $\sigma_{\mathrm{v}}=296 \mathrm{kPa}$

that the sample reached a stable grading where no further crushing was expected to occur. However, these values are bellow the maximum fragmentation fractal dimension (around 2.5-2.6) reported in the literature (Turcotte, 1986; Sammis, 1997; McDowell and Bolton, 1998; McDowell and Daniell, 2001). This is in agreement with the fact that more crushing was produced when increasing the vertical applied stress (Fig. 3).

Figure 6 shows a photograph of selected particles from the samples after the tests. Each column shows particles from samples subjected to different values of angular deformation, while each row shows the size of these particles. It is interesting to see how the original particles fractured into two big particles and some small fragments. Also, it can be noted how some particles lost their angularities as a result of crushing.

\section{DEM ANALYSIS}

The $\mathrm{PFC}^{2 \mathrm{D}}$ program produced by Itasca was used to visualize the evolution of crushing in granular materials under ring shear test conditions. In the $\mathrm{PFC}^{2 \mathrm{D}}$ program, particles are idealized as discs that interact with each other at their contacts. This interaction is mainly governed by three models: the stiffness model, the slip model, and the bonding model (Itasca, Theory and Background, 2002). Only the first two models were used in this simulation. The $\mathrm{PFC}^{2 \mathrm{D}}$ program does not consider particle breakage.

\section{Particle Breakage Criterion}

A subroutine using the FISH language (Itasca, FISH in PFC, 2002) was programmed in order to allow particle breakage. A simplified failure criterion adopted during the simulation considers:

-Only particles with a coordination number equal to or smaller than 3 are able to be broken. Particles subjected to external loads resist them through interparticle contact forces (force chains). These contact forces
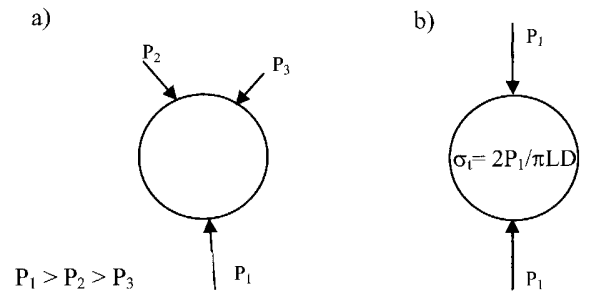

c)

Fig. 7. Idealization of the induced tensile stress and arrangement of the produced fragments

induce tensile stresses in the particles if the coordination number is low (low confinement, coordination number less or equal than 3 for a two-dimensional DEM analysis). These tensile stresses could break the particles (Fig. 7). If the coordination number is high (high confinement, coordination number greater than 3 ), the confinement stresses induced on a particle by their neighbors generates a hydrostatic state of stresses making a tensile failure of the particle less likely (Lade et al., 1996; Tsoungui et al., 1999; Nakata et al., 2001a).

-For those particles having a coordination number smaller than or equal to 3 , the real loading configuration such as the one presented in Fig. 7(a) is assumed to be equivalent to the one obtained in a diametrical compression test such as the Brazilian test, as shown in Fig. 7(b). By using this simplification the induced tensile stress, $\sigma_{\mathrm{t}}$, can be approximated with the expression presented on Fig. 7(b), where $P_{1}$ is the value of the highest contact force acting on the particle, $L$ is the thickness of the disk (unit thickness for the simulated case), and $D$ is the diameter of the disk. The induced tensile stress in Fig. 7(b) could be more severe than the stress induced in Fig. 7(a), but the authors believe that the implemented simplification is justified due to the simplicity in the calculation of the induced tensile stress.

- The tensile strength of a particle having a radius of $1 \mathrm{~mm}$ is predefined as $\sigma_{\text {maxlmm }}=3 \times 10^{6} \mathrm{~Pa}$. This value was arbitrarily chosen by the authors. It represents the strength of the virtual material. By changing this value, the necessary stresses to produce crushing will also change. It is assumed that the tensile strength of a particle with a radius $r, \sigma_{\max }(r)$, is related to $\sigma_{\max 1 \mathrm{~mm}}$ according to the following relationship (where $r$ is expressed in $\mathrm{mm}$ ):

$$
\sigma_{\max }(r)=\sigma_{\max 1 \mathrm{~mm}}[r]^{-1}
$$

In this way, particles with a radius greater than $1 \mathrm{~mm}$ have a tensile strength smaller than $\sigma_{\max 1 \mathrm{~mm}}$, and particles with a radius smaller than $1 \mathrm{~mm}$ have a tensile strength greater than $\sigma_{\text {maximm }}$. Other researchers have reported experimental results in order to describe the change in tensile strength as a function of size for different materials such as quartz fibers and sand grains (Billam, 1971; Nakata et al., 2001b). They found that the experimental results could be described using an 
equation similar in form to Eq. (2). As it is expected, the values of the two constants in Eq. (2) depend on the analyzed material.

-Every particle with a coordination number smaller than or equal to 3 is allowed to break if $\sigma_{\mathrm{t}}>\sigma_{\max }(r)$.

It was assumed that if a particle is fulfilling the previously established failure criterion, it is allowed to break into a group of 8 particles having 3 different sizes, as shown in Fig. 7(c). The breaking of a particle into 8 fragments as shown in Fig. 7 resembles the observed breaking phenomenon in sugar and real aggregates (Lobo-Guerrero and Vallejo, 2005). The number and distribution of particle sizes after breakage could vary depending of the material tested (Takei et al., 2001; Lobo-Guerrero and Vallejo, 2005). Thus, the authors believe for computer efficiency of the model, the use of 8 particles is reasonable. Also, the model used in this study (Fig. 7) considers only one type of crushing, that is particle fragmentation due to tensile stresses. The other type of particle crushing, that is particle abrasion, is not considered by the model used. Since in the model circular particles were used, crushing due to abrasion is less likely to occur.

In order to implement the failure criterion, a new subroutine was programmed using the FISH language. This subroutine automatically checks if a given particle is fulfilling the failure criterion. If it does, the simulation stops, and the particle that broke is automatically deleted and replaced by the set of particles shown in Fig. 7(c). The subroutine does not restrict smaller particles from continuing to break. Thus, particles representing different generations of crushing can coexist inside the sample.

\section{Configuration of the Samples}

The first step was the construction of the virtual ring shear apparatus. First a simulated box having $15 \mathrm{~cm}$ in width and $6 \mathrm{~cm}$ in height was created. The top and bottom walls simulate rough walls having a wave length of $2.5 \mathrm{~cm}$ and a difference in height between peaks of $0.5 \mathrm{~cm}$. Their coefficients of normal and shear stiffness were set to $1 \times 10^{9} \mathrm{~N} / \mathrm{m}$, and their friction coefficients were set to 0.7 . The assumed properties of these walls represent well their roughness and the interaction between the walls and the particles simulating the sugar in the actual ring shear apparatus. Originally these two walls were separated by a vertical distance of $6 \mathrm{~cm}$ before the generation of the particles. Lateral periodic boundaries were programmed by the authors using the FISH language (Itasca, FISH in PFC, 2002). The lateral periodic boundaries and the two horizontal walls represent the frontal surface of an unfolded hollow cylindrical sample enclosed by the ring shear apparatus. Thus, what happens in the frontal surface of a closed cylindrical section can be viewed in its unfolded state as a rectangular window. When using periodic boundaries, particles located at one lateral side of the sample interact with particles located at the opposite lateral side. Particles located at the lateral boundaries experience the contact forces generated by their neighbors located at the opposite side. Moreover, particles are allowed to exit from one side of the sample entering at the same height on the other side. These particles pass from one side to the other conserving their original velocity. Thus, the use of two-dimensional periodic boundaries is a technique used to represent what happens to a granular material in a three-dimensional ring shear apparatus. This technique has been used before by many researcher using DEM codes (Morgan and Boettcher, 1999; Jensen et al., 1999; Jensen et al., 2001; Lang, 2002).

The samples used on the two simulations came from the same original sample. This original sample was created generating 230 circular discs with a radius of $3 \mathrm{~mm}$ and density of $2500 \mathrm{~kg} / \mathrm{m}^{3}$ inside the simulated ring shear. Their positions were randomly generated by the program with no overlaps between discs. The used normal and shear stiffness were set to $1 \times 10^{8} \mathrm{~N} / \mathrm{m}$, and their friction coefficient was set to 0.7 . The values of these properties are similar to those used by Lobo-Guerrero and Vallejo (2005) when simulating the crushable behavior of weak granular materials subjected to direct shear test conditions. The discs were allowed to settle and reorganize under the action of a gravity field $(g=9.8$ $\mathrm{m} / \mathrm{s}^{2}$ ). Thus, the original sample had a loose structure. A vertical force of $1 \times 10^{5} \mathrm{~N}$ was applied by moving downwards the upper wall of the simulated ring shear. This applied force was constant for the remaining part of the first simulation. For the second simulation, the applied force was increased to a value of $1.5 \times 10^{5} \mathrm{~N}$, and was kept constant for the remaining part of that simulation. In both simulations the shear stress was induced by moving the upper wall of the simulated ring shear to the left with a constant velocity of $5 \times 10^{-7} \mathrm{~m} / \mathrm{step}$.

\section{Crushing, Porosity, and Shear Strength Evolution of the} Sample Subjected to a Vertical Force of $1 \times 10^{5} \mathrm{~N}$

Figure 8 shows the applied shear force, the average porosity, and some snap shots at different levels of deformation of the sample subjected to a vertical force of $1 \times 10^{5} \mathrm{~N}$. It can be noted how the applied shear force exhibited a constant value regardless the severe and progressive degradation of the sample. This is in agreement with the experimental results obtained in the sugar samples and those obtained by Coop et al. (2004). An average value for the applied shear force was equal to $0.3 \times 10^{5} \mathrm{~N}$; thus, the average residual friction coefficient was equal to $0.3\left(0.3 \times 10^{5} \mathrm{~N} / 1 \times 10^{5} \mathrm{~N}\right)$.

The porosity of the sample significantly decreased from 0.175 to 0.153 due to particle crushing and particle rearrangement between horizontal deformations of 0 and $5 \mathrm{~cm}$. After this, a very small dilation (porosities between 0.153 to 0.157 ) was produced due to the particle rearrangement between horizontal deformation of $5 \mathrm{~cm}$ and $10 \mathrm{~cm}$. Finally, the sample slightly contract between horizontal deformations of $10 \mathrm{~cm}$ and $15 \mathrm{~cm}$ (porosities of 0.157 and 0.149 respectively). The interaction between contractancy due to particle crushing and dilatancy due to particle rearrangement is far from simple, since both 


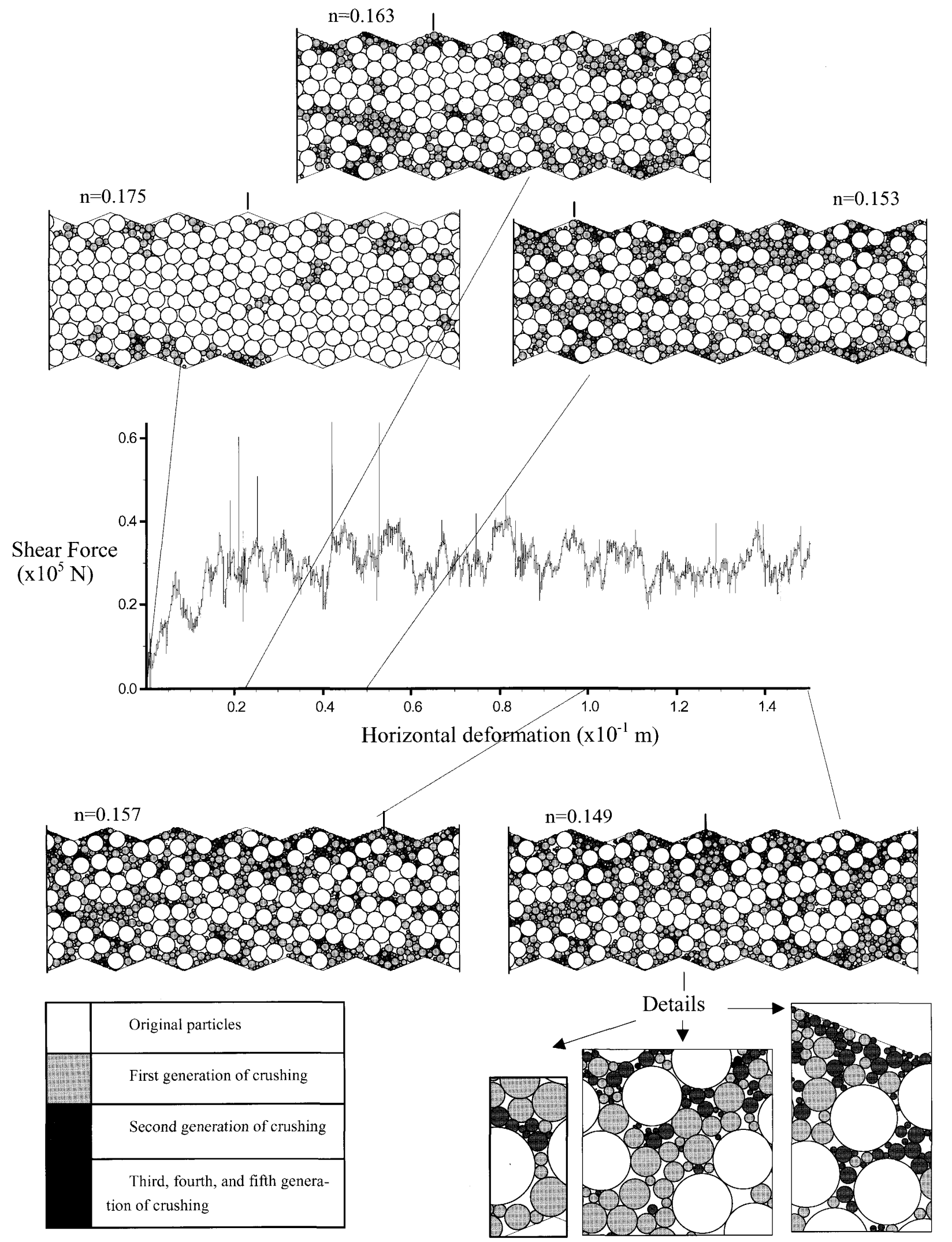

Fig. 8. Crushing, porosity, and shear force evolution of the simulated granular material (vertical force $1 \times 10^{5} \mathrm{~N}$ )

phenomena takes place at the same time. It is complicated to conclude if the sample reached a critical state by only looking the porosities of the sample since an almost constant value of porosity could be the balanced effect between contraction due to crushing and dilation due to particle rearrangement.

It can be observed how different generations of crushing coexist inside the sample, and some of the 


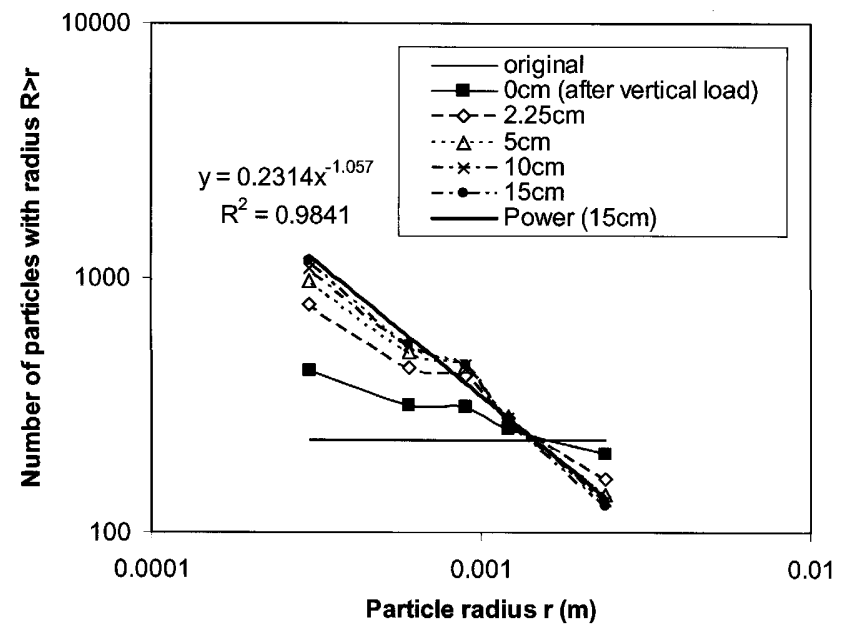

Fig. 9. Evolution of the grain size distribution of the simulated sample (vertical force $1 \times 10^{5} \mathrm{~N}$ )

original particles were surrounded and protected by smaller particles. The grain size distributions of the sample at different values of horizontal deformation were analyzed using the following equation (Turcotte, 1986)

$$
N(R>r)=K(r)^{-D_{\mathrm{i}}}
$$

Where $N(R>r)$ is the number of particles with a radius $R$ (could be other linear dimension) bigger than a given value $r$. $K$ represents a constant and $D_{\mathrm{f}}$ is the previously defined fragmentation fractal dimension. The results are presented in Fig. 9. Also, a power regression line was added to the data corresponding with a horizontal deformation of $15 \mathrm{~cm}$. The obtained fragmentation fractal dimension was equal to 1.057. This value of fragmentation fractal dimension in this two dimensional arrangement corresponds with an equivalent value of 2.057 in a three dimensional case $\left(D_{\mathrm{f} 3 \mathrm{D}}=D_{\mathrm{f} 2 \mathrm{D}}+1\right)$ (McDowell and Daniell, 2001; Sammis, 1997). Thus, the simulated sample achieved a fragmentation fractal dimension equivalent to the maximum value obtained in the laboratory tests on sugar (Fig. 5). Figure 10 shows the evolution of the fragmentation fractal dimension. It shows that the sample presented an almost constant fractal dimension between horizontal deformations of $7.5 \mathrm{~cm}$ and $15 \mathrm{~cm}$. Crushing seems to have ceased at these values of deformation. However, a very small amount of crushing was still occurring in the sample, as is shown in Fig. 10 .

The analysis of the results from the porosities at different values of horizontal deformation and the results from Fig. 10 shows that the sample tended to achieve an almost steady state after a horizontal deformation of $7.5 \mathrm{~cm}$ since the changes in porosity and grain size distribution were very small. Nevertheless, it should be noted that a critical state was not completely achieved since the sample continued to experience a very small amount of crushing even at very large deformations.

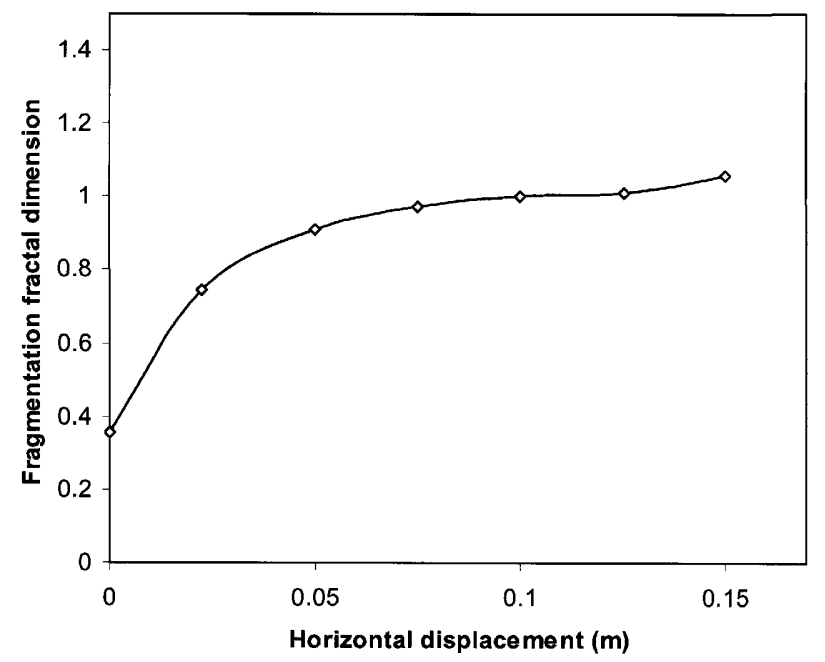

Fig. 10. Evolution of the fragmentation fractal dimension (vertical force $1 \times 10^{5} \mathrm{~N}$ )

Crushing, Porosity, and Shear Strength Evolution of the Sample Subjected to a Vertical Force of $1.5 \times 10^{5} \mathrm{~N}$

Figure 11 shows the applied shear force, the average porosity, and some snap shots at different levels of deformation of the sample subjected to a vertical force of $1.5 \times 10^{5} \mathrm{~N}$. The applied shear force exhibited an almost constant value of $0.45 \times 10^{5} \mathrm{~N}$ regardless of the severe and progressive degradation of the sample. Thus, the average residual friction coefficient was again equal to $0.3(0.45 \times$ $\left.10^{5} \mathrm{~N} / 1.5 \times 10^{5} \mathrm{~N}\right)$. This is in agreement with the results from the first simulation, the experimental results obtained in the sugar samples, and those obtained by Coop et al. (2004).

The porosity of the sample significantly decreased from 0.166 to 0.139 due to particle crushing and particle rearrangement between horizontal deformations of 0 and $5 \mathrm{~cm}$. After this, the porosity continued to decrease but at a very small rate (from 0.139 to 0.130 in $5 \mathrm{~cm}$ of horizontal deformation). It seems that the higher applied vertical force precluded the occurrence of dilatancy. Figure 12 shows the results of applying Eq. (3) to the grain size distributions of the sample at different values of horizontal deformation. A maximum value of fragmentation fractal dimension equal to 1.1484 (Equivalent to 2.1484 in 3D) was achieved by the sample at the end of the simulation. More crushing occurred during this second simulation than during the first one. This is reflected in the fact that the fragmentation fractal dimension was higher than the one obtained during the first simulation (2.1484 vs. 2.057). This is in agreement with the laboratory results presented in Fig. 3.

Figure 13 shows the evolution of the fragmentation fractal dimension during the second simulation. It can be noted that the sample achieved an almost constant fragmentation fractal dimension at a horizontal displacement of $5 \mathrm{~cm}$. A comparison of Figs. 13 and 11 shows that the sample reached an almost steady state at a horizontal deformation of $5 \mathrm{~cm}$ since the changes in porosity and fragmentation fractal dimension were considerably small 


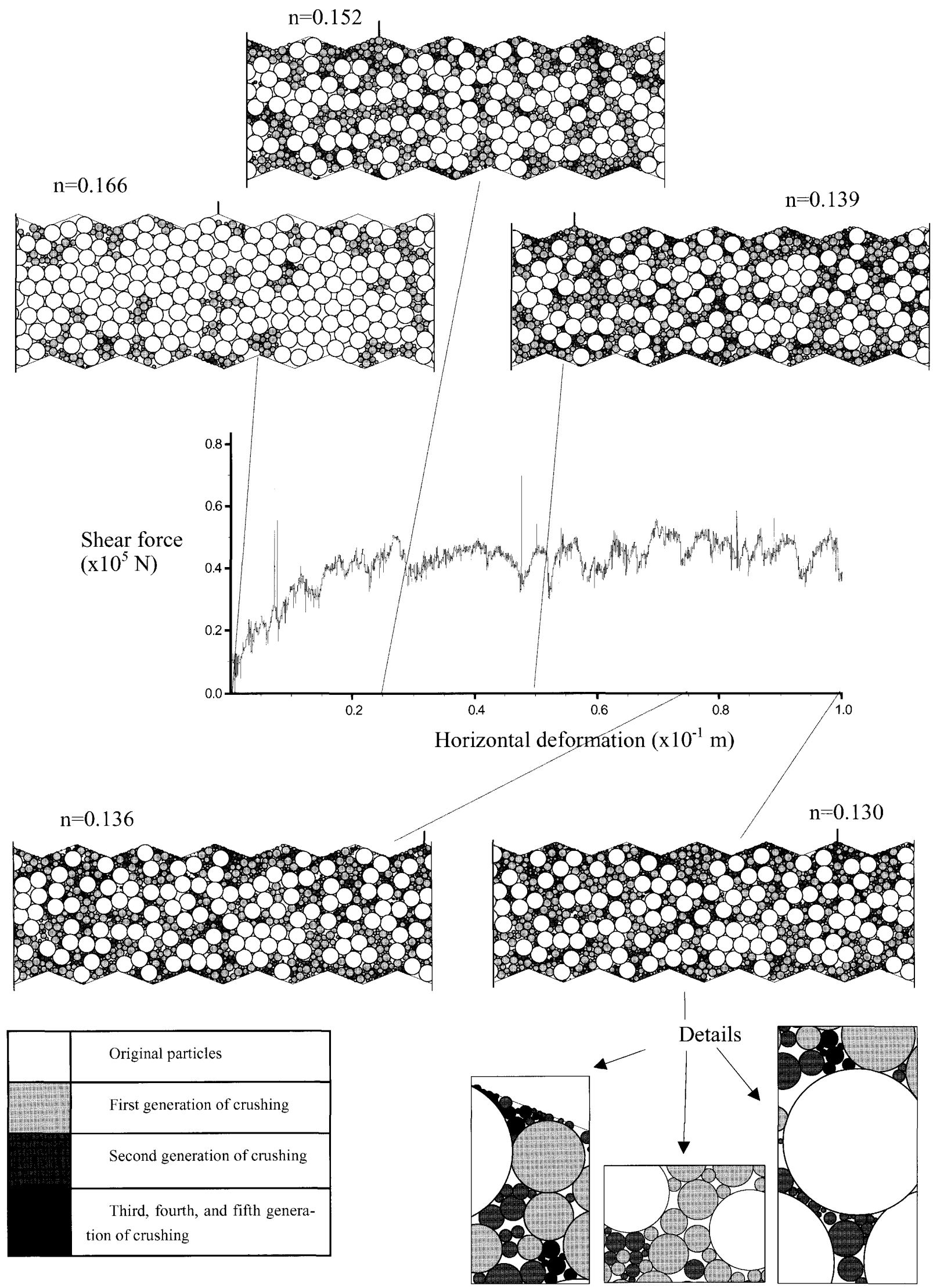

Fig. 11. Crushing, porosity, and shear force evolution of the simulated granular material (vertical force $1.5 \times 10^{5} \mathrm{~N}$ ) 


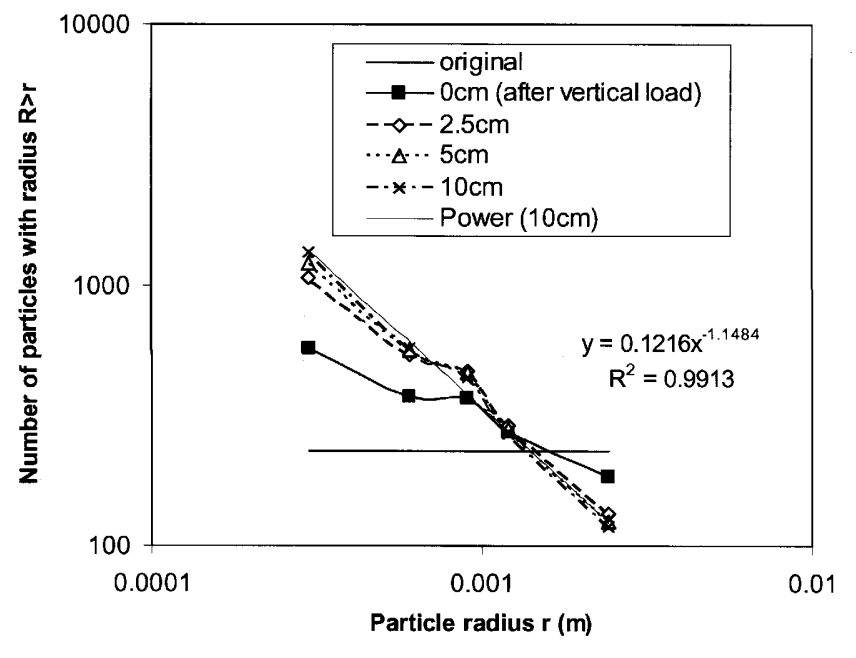

Fig. 12. Evolution of the grain size distribution of the simulated sample (vertical force $1.5 \times 10^{5} \mathrm{~N}$ )

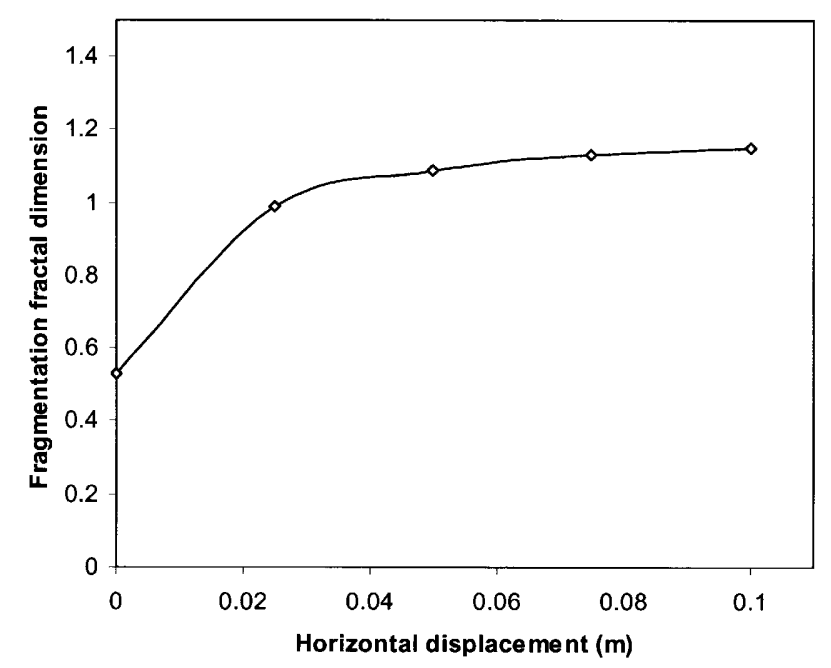

Fig. 13. Evolution of the fragmentation fractal dimension (vertical force $1.5 \times 10^{5} \mathrm{~N}$ )

after this deformation. Nevertheless, it should be noted that a critical state was not completely achieved since the sample continue to experience a very small amount of crushing even at very large deformations.

\section{CONCLUSIONS}

The evolution of crushing in a granular material subjected to a ring shear test was studied using laboratory tests and DEM simulations. The ring shear tests were carried out in the laboratory using sugar. The residual friction coefficient of this material maintained a constant value regardless of the severe degradation of the particles. This degradation was induced by increasing the angular deformation or increasing the applied vertical stress. Moreover, it was found that the grain size distribution of the original uniform material evolved toward a fractal distribution of sizes.

The results from the DEM simulations confirmed those from the laboratory tests and provided also a visualization of the crushing evolution. DEM is a valuable tool that can be used to understand complex phenomena that occur in the evolution of crushing such as the interaction between sample contraction generated by particle breakage and dilation due to particle rearrangement.

\section{ACKNOWLEDGMENTS}

This work was supported by Grant No. CMS-0301815 to the University of Pittsburgh from the National Science Foundation, Washington, D.C. This support is gratefully acknowledged.

\section{REFERENCES}

1) Billam, J. (1971): Some aspects of the behaviour of granular materials at high pressures, Stress-strain Behaviour of Soils: Proc. Roscoe Memorial Symposium, Cambridge University, 69-80.

2) Coop, M. R., Sorensen, K. K., Bodas Freitas, T. and Georgoutsos, G. (2004): Particle breakage during shearing of carbonate sand, Geotechnique, 54(3), 157-163.

3) Cundall, P. A. and Strack, O. D. L. (1979): A discrete numerical model for granular assemblies, Geotechnique, 29(1), 47-65.

4) Itasca Consulting Group, Inc. (2002): $P F C^{2 D}$ (Particle Flow Code in Two Dimensions) version 3.0: Theory and Background, and FISH in PFC.

5) Jensen, R. P., Bosscher, P. J., Plesha, M. E. and Edil, T. B. (1999): DEM simulation of granular media-structure interface: effects of surface roughness and particle shape, Int. J. Num. Anal. Meth. Geomech., 23, 531-547.

6) Jensen, R. P., Plesha, M. E., Edil, T. B., Bosscher, P. J. and Kahla, N. B. (2001): DEM simulation of particle damage in granular media-structure interfaces, Int. J. Geomech., 1(1), 21-39.

7) Lade, P. V., Yamamuro, J. A. and Bopp, P. A. (1996): Significance of particle crushing in granular materials, J. Geotech. Engrg., ASCE, 122(4), 309-316.

8) Lang, R. A. (2002): Numerical simulation of comminution in granular materials with an application to fault gouge evolution, Master Thesis, Texas A\&M University.

9) Lobo-Guerrero, S. and Vallejo, L. E. (2004): Modeling of material crushing in granular road bases, Proc. UNBAR VI Conf., Pavements Unbound, University of Nottingham, England, 33-41.

10) Lobo-Guerrero, S. and Vallejo, L. E. (2005): Crushing a weak granular material: experimental-numerical analyses, Geotechnique, 55(3), 245-249.

11) Luzzani, L. and Coop, M. R. (2002): On the relationship between particle breakage and the critical state of sands, Soils and Foundations, 42(2), 71-82.

12) Mandl, G., Jong, L. N. J. and Maltha, A. (1977): Shear zones in granular material, Rock Mechanics, 9, 95-144.

13) McDowell, G. R. and Bolton, M. D. (1998): On the micromechanics of crushable aggregates, Geotechnique, 48(5), 667-679.

14) McDowell, G. R. and Daniell, C. M. (2001): Fractal compression of soil, Geotechnique, 51(2), 173-176.

15) McDowell, G. R. and Humphreys, A. (2002): Yielding of granular materials, Granular Matter, (4), 1-8.

16) McDowell, G. R. and Khan, J. J. (2003): Creep of granular materials, Granular Matter, (5), 115-120.

17) Morgan, J. K. (1999): Numerical simulations of granular shear zones using the distinct element method, 2. Effects of particle size distribution and interparticle friction on mechanical behavior, $J$. Geophysical Research, 104(B2), 2721-2732.

18) Morgan, J. K. and Boettcher, M. S. (1999): Numerical simulations of granular shear zones using the distinct element method, 1. Shear zone kinematics and the micromechanics of localization, $J$. Geophysical Research, 104(B2), 2703-2719. 
19) Nakata, Y., Hyodo, M., Hyde, A. F. L., Kato, Y. and Murata, H. (2001a): Microscopic particle crushing of sand subjected to high pressure one-dimensional compression, Soils and Foundations, 41(1), 69-82.

20) Nakata, Y., Kato, Y., Hyodo, M., Hyde, A. F. L. and Murata, H. (2001b): One-dimensional compression behaviour of uniformly graded sand related to single particle crushing strength, Soils and Foundations, 41(2), 39-51.

21) Sammis, C. G. (1997): Fractal fragmentation and frictional stability in granular materials, IUTAM Symp. Mechanics of Granular and Porous Materials, 23-34.

22) Takei, M., Kusakabe, O. and Hayashi, T. (2001): Time dependent behavior of crushable materials in one-dimensional compression tests, Soils and Foundations, 41(1), 97-121.

23) Tsoungui, O., Vallet, D. and Charmet, J. C. (1999): Numerical model of crushing of grains inside two-dimensional granular materials, Powder Technology, 105, 190-198.

24) Turcotte, D. L. (1986): Fractals and fragmentation, J. Geophysical Research, 91(B2), 1921-1926.

25) Tyler, S. W. and Wheatcraft, S. W. (1992): Fractal scaling of soil particle-size distribution analysis and limitations, Soil Science Society of America J., 56(2), 47-67.

26) Vallejo, L. E. (2003): Crushing of granular bases: fractal and laboratory analyses, Proc. I Iberoamerican Symp. Pavement Engineering/XIV Colombian Symp. Pavement Engineering, Popayan Colombia, CD-ROM. 